\title{
Constraint-Based Modeling in Systems Biology
}

\author{
Alexander Bockmayr \\ DFG-Research Center MATHEON, \\ FB Mathematik und Informatik, \\ Freie Universität Berlin \\ Arnimallee 6. 14195 Berlin. Germany \\ Alexander.Bockmayr@fu-berlin.de
}

\begin{abstract}
Systems biology is a new interdisciplinary research field that has received considerable attention in recent years. While traditional molecular biology studies the various components of a biological system (e.g. genes, RNAs, proteins) in isolation, systems biology aims to understand how these components interact in order to perform complex biological functions. A variety of mathematical and computational methods is currently being used to model and analyze biological systems, ranging from continuous, stochastic, and discrete to various hybrid approaches.

The idea of constraint-based modeling in systems biology is to describe a biological system by a set of constraints, i.e., by pieces of partial information about its structure and dynamics. Using constraint-based reasoning one may then draw conclusions about the possible system behaviors.

In this talk, we will focus on constraint-based modeling techniques for regulatory networks starting from the discrete logical formalism of René Thomas. In this framework, logic and constraints arise at two different levels. On the one hand, Boolean or multi-valued logic formulae provide a natural way to represent the structure of a regulatory network, which is given by positive and negative interactions (i.e., activation and inhibition) between the network components. On the other hand, temporal logic formulae (e.g. CTL) may be used to reason about the dynamics of the system, represented by a state transition graph or Kripke model.

Emphasis will be on non-deterministic modeling of the network dynamics and model checking techniques for network inference. We will also discuss how to include additional temporal constraints on time delays in a hybrid discrete-continuous modeling framework.
\end{abstract}

